\title{
HIGHLIGHTS
}

TESTICULAR CANCER

\section{Surveillance or biopsy for men with testicular microlithiasis?}

A systematic review of data collected from adults with testicular microlithiasis should help to resolve the confusion regarding the optimal management strategy for this increasingly common condition.

According to senior author Min-Han Tan "the relationship between testicular microlithiasis and testicular germ

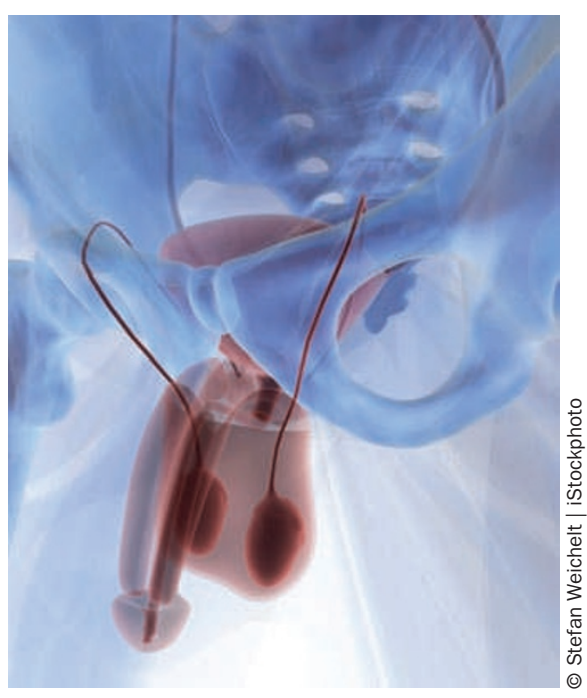

cell tumor (TGCT) has always [been] controversial." Tan's Singapore-based team sought to more-clearly define the association by systematically analyzing data from 33 studies. The investigators conclude that the association between microlithiasis and TGCT depends on clinical context.

Incidental detection of microliths in healthy asymptomatic men was not associated with an increased likelihood of developing TGCT. By contrast, patients with testicular microliths plus risk factors for TGCT (such as, subfertility, cryptorchidism, or previous orchiectomy for testicular malignancy) were 8.5 times more likely to develop a tumor.

Ever more cases of microlithiasis are coming to light-a function of the increasingly widespread use of highresolution scrotal ultrasound. There are no validated global standards on which to base follow up of men with this condition. As such, practice varies greatly between regions. On the basis of their study, Tan and colleagues assert that management of men with testicular microlithiasis should be dictated by the presence or absence of concurrent risk factors for TGCT. Their paper, published in Cancer, includes an algorithm outlining their recommendations, which escalate from no follow-up, through self-exam plus follow-up, to biopsy.

"An aggressive approach towards testicular biopsy in patients with microlithiasis and TGCT [risk factors] is likely to detect more patients with contralateral precursor lesions. However, given that clinically-manifested testicular cancer remains a highly curable disease, it is uncertain that early diagnosis using an invasive maneuver will result in improved outcomes over a surveillance strategy" concludes Tan.

Suzanne J. Farley

Original article Tan, I. B. et al. Testicular microlithiasis predicts concurrent testicular germ cell tumors and intratubular germ cell neoplasia of unclassified type in adults. Cancer doi:10.1002/cncr.25231 